\title{
Circ_0001421 facilitates glycolysis and lung cancer development by regulating miR- 4677-3p/CDCA3
}

\author{
Koudong Zhang ${ }^{1}$, Hang Hu${ }^{1}$, Juan $\mathrm{Xu}^{1}$, Limin Qiu ${ }^{2}$, Haitao $\mathrm{Chen}^{3}$, Xingzhi Jiang ${ }^{1}$ and Yongqian Jiang ${ }^{*}$
}

\begin{abstract}
Background: Lung cancer $(\mathrm{LC})$ is a malignant tumor originating in the bronchial mucosa or gland of the lung. Circular RNAs (circRNAs) are proved to be key regulators of tumor progression. However, the regulatory effect of circ_0001421 on lung cancer tumorigenesis remains unclear.

Methods: The expression levels of circ_0001421, microRNA-4677-3p (miR-4677-3p) and cell division cycle associated 3 (CDCA3) were detected by quantitative real-time polymerase chain reaction (qRT-PCR). Methyl thiazolyl tetrazolium (MTT), Transwell and Tumor formation assays were performed to explore the role of circ_0001421 in LC. Glucose consumption and lactate production were examined by a Glucose assay kit and a Lactic Acid assay kit. Western blot was utilized to examine the protein levels of Hexokinase 2 (HK2) and CDCA3. The interaction between miR-4677-3p and circ_0001421 or CDCA3 was confirmed by dual-luciferase reporter assay.
\end{abstract}

Results: Circ_0001421 was increased in LC tissues and cells, and knockdown of circ_0001421 repressed cell proliferation, migration, invasion and glycolysis in vitro. Meanwhile, circ_0001421 knockdown inhibited LC tumor growth in vivo. Mechanistically, circ_0001421 could bind to miR-4677-3p, and CDCA3 was a target of miR-4677-3p. Rescue assays manifested that silencing miR-4677-3p or CDCA3 overexpression reversed circ_0001421 knockdownmediated suppression on cell proliferation, migration, invasion and glycolysis in LC cells.

Conclusion: Circ_0001421 promoted cell proliferation, migration, invasion and glycolysis in LC by regulating the miR-4677-3p/CDCA3 axis, which providing a new mechanism for LC tumor progression.

Keywords: Lung cancer, circ_0001421, miR-4677-3p, CDCA3

\section{Background}

Lung cancer (LC) is a malignant tumor with the highest morbidity and mortality in worldwide [1,2]. According to histopathological classification, LC included non-small cell lung cancer (NSCLC) and small cell lung cancer. The 5year survival rate of LC patients is only $17.7 \%$, mainly on account of the rapid tumor metastasis and local recurrence [3]. Besides, the 5-year survival rate of patients with

\footnotetext{
* Correspondence: claudyzkd@163.com

'Department of Respiratory and Critical Medicine, The Fourth Affiliated Hospital of Nantong University; The First People's Hospital of Yancheng, No.66 Renmin South Road, Yancheng 224000, Jiangsu, China

Full list of author information is available at the end of the article
}

early-stage LC is significantly higher than that of advanced LC patients [4]. Hence, it is necessary to dive deep into the underlying mechanism of lung cancer tumorigenesis and progression.

Circular RNA (circRNA) is a special form of non-coding RNA (ncRNA) that form a loop with jointed 3 ' heads and $5^{\prime}$ tails [5]. CircRNAs have received attention due to their conservation and tissue specific expression in living beings [6]. The regulatory mechanism of circRNAs has been further investigated, and some circRNAs play regulatory roles by serving as sponges to adsorb microRNAs (miRNAs) [7]. A number of circRNAs were involved in cancer

(c) The Author(s). 2020 Open Access This article is licensed under a Creative Commons Attribution 4.0 International License, which permits use, sharing, adaptation, distribution and reproduction in any medium or format, as long as you give appropriate credit to the original author(s) and the source, provide a link to the Creative Commons licence, and indicate if changes were made. The images or other third party material in this article are included in the article's Creative Commons licence, unless indicated otherwise in a credit line to the material. If material is not included in the article's Creative Commons licence and your intended use is not permitted by statutory regulation or exceeds the permitted use, you will need to obtain permission directly from the copyright holder. To view a copy of this licence, visit http://creativecommons.org/licenses/by/4.0/ The Creative Commons Public Domain Dedication waiver (http://creativecommons.org/publicdomain/zero/1.0/) applies to the data made available in this article, unless otherwise stated in a credit line to the data. 
development. For example, circNRIP1 boosted cell growth, migration and invasion through sponging miR149-5p in gastric cancer [8]. Silencing circDENND4C repressed breast cancer cell glycolysis and metastasis by sponging miR-200b/c under hypoxia [9]. The effects of circRNAs on LC have also been reported. CircFGFR1 could elevate cell proliferation and invasion by targeting miR-381-3p in NSCLC [10]. CircRNA SMARCA5 played an inhibition role in the progression of LC by acting as miR-19b-3p sponge [11]. Circular RNA, circ_0001421 (circ-SEC31A) was upregulated in NSCLC and its high expression predicts unfavorable prognoses [12]. However, the role of circ_0001421 in lung cancer tumorigenesis and progression was needed to be elucidated further.

MiRNAs are also ncRNA molecules with $18 \sim 25$ nucleotides in length, and they play a biological role by targeting the downstream mRNAs [13]. As reported, miR196b-5p promoted tumor development in NSCLC by targeting TSPAN12 and GATA6 [14]. MiR-212-5p enhanced tumor growth by regulating Id3 in lung adenocarcinoma cells [15]. MiR-4677-3p was found to be decreased in lung adenocarcinoma tissue samples, and lncRNA TTN-AS1 elevated cell migration and invasion by acting as miR4677-3p sponge [16], whereas the interaction between miR-4677-3p and circ_0001421 was not studied in LC.

Cell division cycle associated protein-3 (CDCA3) modulates cell cycle processes, also called as trigger of mitosis entry 1 , and it was identified to play a procarcinogenic role in gastric cancer [17], colorectal cancer [18] and acute myeloid leukemia [19]. However, the function of CDCA3 in LC has been little studied, and its effect is not fully understood.

Here, we investigated the expression of circ_0001421 in LC and its effects on cell proliferation, migration, invasion and glycolysis. Additionally, we appraised the interaction between circ_0001421 and miR-4677-3p, as well as the downstream genes. In conclusion, the aim of this study was to explore the role of circ_0001421 in LC progression.

\section{Materials and methods}

\section{Clinical tissues and cell}

The 48 paired lung cancer tissues and adjacent normal tissues were collected from lung cancer patients, who were diagnosed in The Fourth Affiliated Hospital of Nantong University; The First People's Hospital of Yancheng from June 2016 to December 2018. The samples were immediately transferred to liquid nitrogen for further research. Written informed consents were obtained from these LC patients. And this study was reviewed and approved by The Fourth Affiliated Hospital of Nantong University; The First People's Hospital of Yancheng. Table 1 presents the clinical characteristics.
Human LC cells (A549 and H1299) bought from American Type Culture Collection (ATCC, Manassas, VA, USA) and human normal bronchial epithelial cells (16HBE) bought from Procell (Wuhan, China) were maintained in Dulbecco's Modified Eagle Medium (DMEM, Invitrogen, Carlsbad, CA, USA) containing $10 \%$ fetal bovine serum (FBS) at $37^{\circ} \mathrm{C}$ with $5 \% \mathrm{CO}_{2}$.

\section{Transfection}

Small interfering RNA targeting circ_0001421 (si-circ_ 0001421， 5'-CCGGCTCTGGAGTTCTGATTGCATTC TCGAGTGCAATCAGAACTCCAGAGTTTTTTTG-3') and negative control (si-NC, $5^{\prime}$-UUCUCCGAACGUGU CACUTT-3'), miR-4677-3p mimic (miR-4677-3p, 5' UCAUCAAGAAACCAGAGUGUCU-3'), mimic NC (miR-NC, 5'-GAAAUGUACUUGAGCGUGGAGAC-3'), miR-4677-3p inhibitor (anti-miR-4677-3p, 5'-GUCUCC ACGCUCAAGUACAUUUC-3'), inhibitor NC (anti-miRNC, 5' -CUAAAACCGGCCGUACGGCGUU-3'), shorthairpin against circ_0001421 (sh-circ_0001421, 5'-CCGG GCTAAGATTCTCTGCTCCAATCTCGAGATTGGAG

Table 1 Correlation between hsa_circ_0001421 expression and clinicopathological characteristics in lung cancer

\begin{tabular}{|c|c|c|c|}
\hline Clinical parameter & $\begin{array}{l}\text { Hsa_circ_0001421 } \\
\text { High }(n=24)\end{array}$ & $\begin{array}{l}\text { Hsa_circ_0001421 } \\
\text { Low }(n=24)\end{array}$ & $P$ \\
\hline \multicolumn{4}{|l|}{ Age (years) } \\
\hline$\leq 60$ & 9 & 11 & 0.107 \\
\hline$>60$ & 15 & 13 & \\
\hline \multicolumn{4}{|l|}{ Gender } \\
\hline Male & 14 & 12 & 0.116 \\
\hline Female & 10 & 12 & \\
\hline \multicolumn{4}{|l|}{ Histological grade } \\
\hline Low or undiffer & 10 & 12 & 0.155 \\
\hline Middle or high & 14 & 12 & \\
\hline \multicolumn{4}{|l|}{ TNM stages } \\
\hline land II & 15 & 16 & $0.018^{*}$ \\
\hline III and IV & 9 & 8 & \\
\hline \multicolumn{4}{|l|}{ Size } \\
\hline$\leq 5 \mathrm{~cm}$ & 8 & 7 & $0.015^{*}$ \\
\hline$>5 \mathrm{~cm}$ & 16 & 17 & \\
\hline \multicolumn{4}{|l|}{ Invasion depth } \\
\hline $\mathrm{T} 1$ and $\mathrm{T} 2$ & 14 & 19 & $0.033^{*}$ \\
\hline $\mathrm{T} 3$ and $\mathrm{T} 4$ & 25 & 12 & \\
\hline \multicolumn{4}{|l|}{ Lymphatic metastasis } \\
\hline Yes & 11 & 10 & 0.153 \\
\hline No & 13 & 14 & \\
\hline \multicolumn{4}{|l|}{ Distant metastasis } \\
\hline Yes & 12 & 11 & 0.188 \\
\hline No & 12 & 13 & \\
\hline
\end{tabular}


CAGAGAATCTTAGCTTTTTTG-3') and control (shNC, 5'-UUCUCCGAACGUGUCACGUTT-3') were acquired from GenePharma (Shanghai, China). Overexpression vector Vector-CDCA3 (CDCA3) and control (Vector) were acquired from GenePharma. Lipofectamine 3000 (Invitrogen) was employed to conduct cell transfection in this study.

\section{Quantitative real-time polymerase chain reaction (qRT- PCR)}

The RNA was extracted from LC tissues and cells via TRIzol (Invitrogen), and then reversely transcribed to complementary DNA (cDNA) through the PrimeScript ${ }^{\mathrm{m}}$ strand cDNA synthesis kit (Takara, Dalian, China). The reaction of qRT-PCR was carried out by a Power SYBRTM Green PCR Master Mix (Thermo Fisher Scientific, Waltham, MA, USA) on an ABI 7900 system (Thermo Fisher Scientific). U6 and glyceraldehyde-3phosphate dehydrogenase (GAPDH) were used as the internal controls for miRNA and circRNA or mRNA, respectively. Primer sequences were listed as follows: circ_0001421, F: 5' -TCTCTGGAGTTCTGATTGCAGG TGG-3' and R: 5'-TGCTAGGTAAATGGGGTGATTC TGG-3'; miR-4677-3p, F: 5'-CTGTGAGACCAAAGAA CTACTCGC-3' and R: 5' -CTCTACAGCTATATTGCC AGCCAC-3'; CDCA3, F: 5'-GGACCCTGAGACTC CCAGAT-3' and R: 5'-GCCGCTTACCCTGTCGTAG3'; GAPDH, F: 5'-CCATTTGCAGTGGCAAAG-3' and R: 5'-CACCCCATTTGATGTTAGTG-3'; and U6, F: 5'-GCTTCGGCAGCACATATACTAAAAT-3' and R: 5'-TACTGTGCGTTTAAGCACTTCGC-3'.

\section{Cell proliferation detection}

The proliferation of A549 and H1299 cells was determined through the Methyl thiazolyl tetrazolium (MTT, Sigma-Aldrich, St. Louis, MO, USA) reagent. Cells were inoculated into the 96-well plates. $10 \mu \mathrm{L}$ MTT was added into the cells. Then, $100 \mu \mathrm{L}$ Dimethyl sulfoxide (DMSO, Sigma-Aldrich) was added to the cells. The absorbance at $490 \mathrm{~nm}$ was detected at $0 \mathrm{~h}, 24 \mathrm{~h}, 48 \mathrm{~h}$ and $72 \mathrm{~h}$ by a microplate reader (Bio-Rad, Hercules, CA, USA).

\section{Transwell assay}

Transwell assay with the chamber was performed to assess cell migration (uncoated Matrigel) and invasion (coated Matrigel, Thermo Fisher Scientific). Transfected LC cells suspended in serum-free medium were seeded into the top chambers, and $600 \mu \mathrm{L}$ medium with $10 \%$ FBS was added into the lower chamber. $24 \mathrm{~h}$ later, cells on the lower surface of chambers were fixed by methanol and then stained with $0.1 \%$ crystal violet for $20 \mathrm{~min}$. Finally, a microscope was used to quantify the number of migrated or invaded cells.

\section{Glucose consumption and lactate production analysis}

The transfected A549 and H1299 cells were harvested at $48 \mathrm{~h}$ post-transfection, and the cell culture medium were collected. Then, the concentration of glucose in cell culture medium was examined by a Glucose assay kit (Sigma-Aldrich, St. Louis, MO, USA), and lactate concentration was determined by a Lactic Acid assay kit (Seebio, Shanghai, China).

\section{Western blot}

Proteins were extracted from cell and tissue lysates using RIPA buffer (Invitrogen), and then separated by sodium dodecyl sulfate polyacrylamide gel electrophoresis (SDSPAGE). Subsequently, samples were transferred onto polyvinylidene fluoride (PVDF, Millipore, Billerica, MA, USA) membranes. $5 \%$ skim milk powder was used to block these membranes for $2 \mathrm{~h}$. Then, the bands were incubated with the primary antibodies against Hexokinase 2 (HK2, 1:1000, Abcam, Cambridge, MA, USA), CDCA3 $(1: 1000$, Abcam $)$ or GAPDH $(1: 2000$, Abcam $)$ at $4{ }^{\circ} \mathrm{C}$ overnight. The next day, the membranes were mixed with the secondary antibodies (1:4000, Abcam) for $1 \mathrm{~h}$. The immunoblots were detected with the enhanced chemiluminescence reagent (Millipore).

\section{Dual-luciferase reporter assay}

To affirm the interaction between miR-4677-3p and circ_0001421 or CDCA3, the wild-type (WT) sequences of circ_0001421 contained miR-4677-3p binding sites or its mutant (MUT) were cloned into the pmirGLO vector (Promega, Madison, WI, USA) to form circ_0001421WT or circ_0001421-MUT reporter vector. CDCA33'UTR-WT and CDCA3-3'UTR-MUT were constructed in the same way. These reporter vectors were cotransfected into cells with miR-4677-3p or miR-NC by using Lipofectamine 3000. $48 \mathrm{~h}$ later, the luciferase activity was determined by a Dual-Luciferase Reporter assay system (Promega).

\section{Xenograft tumors in nude mice}

Twelve male nude BALB/c mice (20-22 g, 4 weeks) were purchased from Shanghai Laboratory Animal Center (SLAC, Shanghai, China). A549 cells transfected with shcirc_0001421 or sh-NC were injected into the nude mice. Tumor volume was measured once a week. Five weeks later, all the mice were euthanatized with an overdose of ether, and tumor weight was measured. Western blot and qRT-PCR were performed to examine the expression of CDCA3 and circ_0001421 or miR-4677-3p, respectively.

\section{Statistical analysis}

Data were displayed as the mean \pm standard deviation (SD). The difference of two groups was evaluated by Wilcoxon test, and one-way analysis of variance 

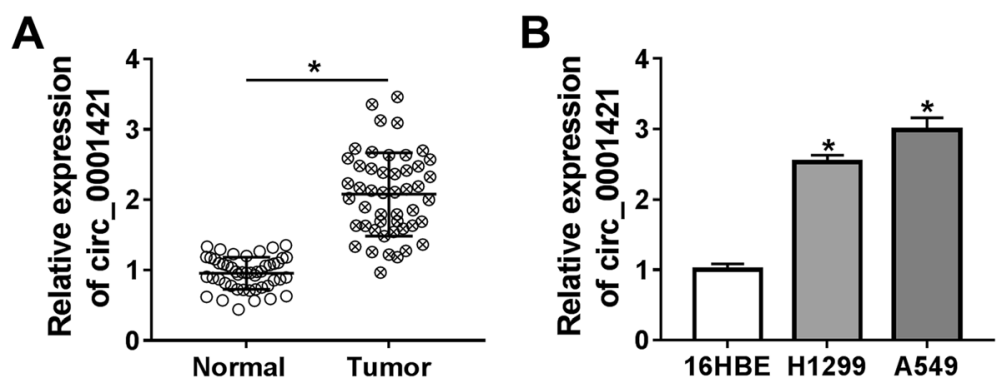

Fig. 1 Circ_0001421 was overexpressed in LC tissues and cells. a Circ_0001421 expression in tumor and adjacent normal tissues was detected by qRT-PCR. $\mathbf{b}$ qRT-PCR was performed to examine circ_0001421 expression in LC cell lines (A549 and H1299) and normal human bronchial epithelial cells $(16 \mathrm{HBE}) .{ }^{*} P<0.05$

(ANOVA) was applied for more than two groups. $P<$ 0.05 was considered as statistically distinct. Each test was repeated at least three times.

\section{Result}

Circ_0001421 was overexpressed in LC tissues and cells Firstly, circ_0001421 expression in tumor and normal tissues of 48 LC patients was measured by qRT-PCR. The results showed that circ_0001421 expression in tumor tissues was significantly higher than that in normal tissues (Fig. 1a). Additionally, circ_0001421 was overexpressed in LC cell lines, including A549 and H1299 cells, compared to 16HBE cells (Fig. 1b).

\section{Knockdown of circ_0001421 repressed LC cell} proliferation, migration, invasion and glycolysis in vitro Subsequently, several experiments were performed to explore the function of circ_0001421 in LC. As shown in

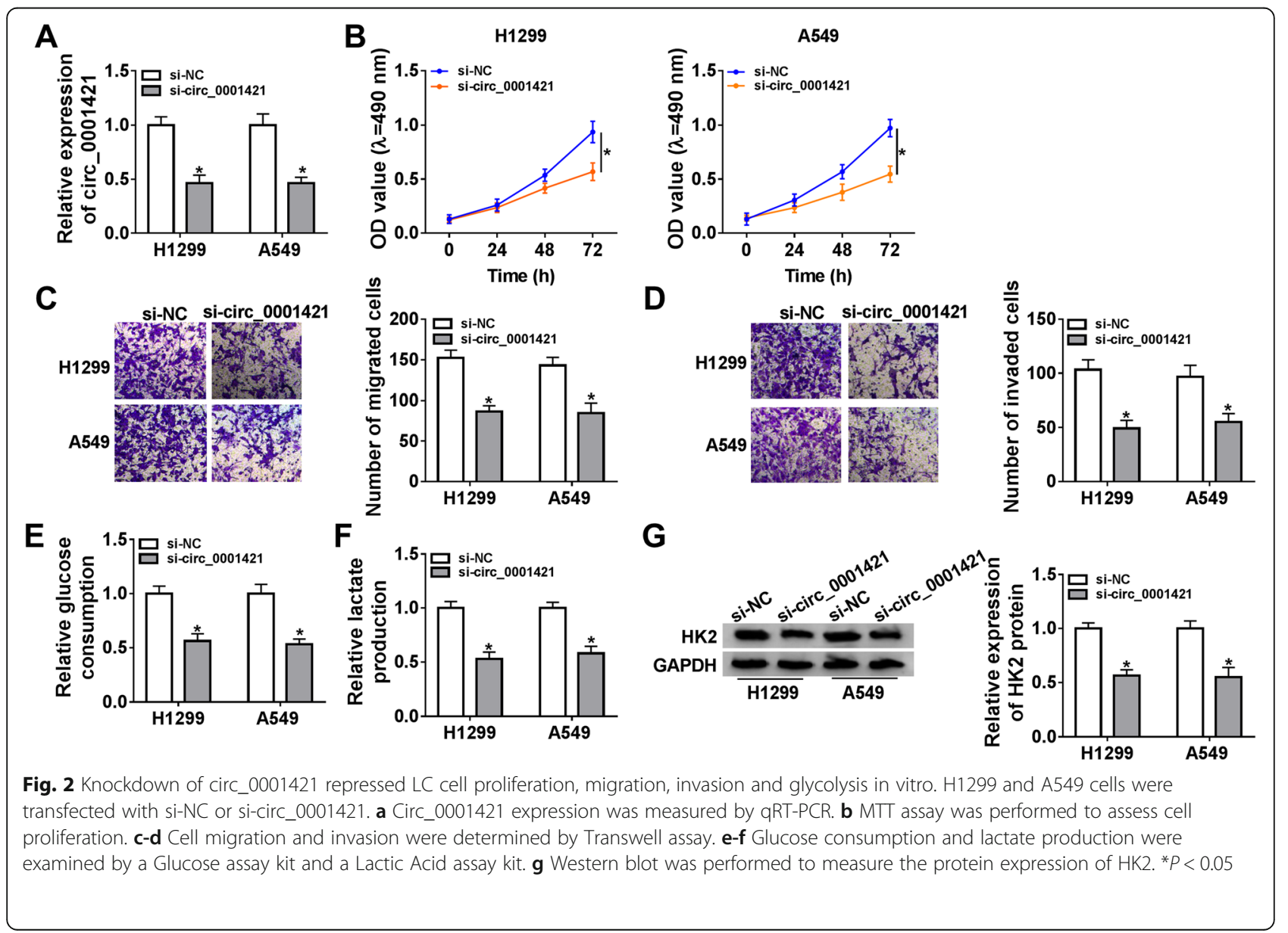


Fig. 2a, the lower expression of circ_0001421 was found in A549 and H1299 cells by transfection of sicirc_0001421 relative to that in si-NC-transfected cells. MTT assay demonstrated that circ_0001421 inhibition retarded proliferation in A549 and H1299 cells comparison with the control group (Fig. 2b). Transwell assay results manifested that circ_0001421 knockdown notably curbed cell migration and invasion of A549 and H1299 cells (Fig. 2c-d). Glucose consumption and lactate production assays were performed to assess the effect of circ_0001421 on glycolysis. Circ_0001421 knockdown significantly reduced glucose consumption and lactate production in A549 and H1299 cells (Fig. 2e-f). Meanwhile, HK2 protein expression in si-circ_0001421 transfected A549 and H1299 cells was declined (Fig. 2g). Collectively, these results supported that circ_0001421 promoted in LC cell proliferation, migration, invasion, glycolysis, and functioned as an oncogenic circRNA.

\section{Circ_0001421 could act as a sponge for miR-4677-3p}

To investigate the molecular mechanism of circ 0001421 regulating the progression of LC cells, Starbase
3.0 was employed to screen the target miRNAs sponged by circ_0001421. The results showed that there were binding sites between circ_0001421 and miR-4677-3p (Fig. 3a), and the relationship between them was then confirmed by dual-luciferase reporter assay. The expression of miR-4677-3p was increased in A549 and H1299 cells by transfection of miR-4677-3p (Fig. 3b). Overexpression of miR-4677-3p drastically degraded the luciferase activity of circ_0001421-WT, but did not distinctly reduce the luciferase activity of circ_0001421-MUT in A549 and H1299 cells (Fig. 3c). Then, we examined miR-4677-3p expression in LC tissues by qRT-PCR. As displayed in Fig. 3d, miR-4677-3p expression was downregulated in tumor tissues compared to normal tissues. In addition, miR-4677-3p expression was exceptionally decreased in A549 and H1299 cells (Fig. 3e). The correlation between circ_0001421 and miR-4677-3p expression in 48 LC patients was then analyzed and a significant negative correlation between them was observed (Fig. 3f). We also found that knockdown of circ 0001421 up-regulated miR-4677-3p expression in A549 and H1299 cells (Fig. 3g). We concluded that circ 0001421 could target miR-4677-3p to negatively regulate miR-4677-3p expression in LC cells.
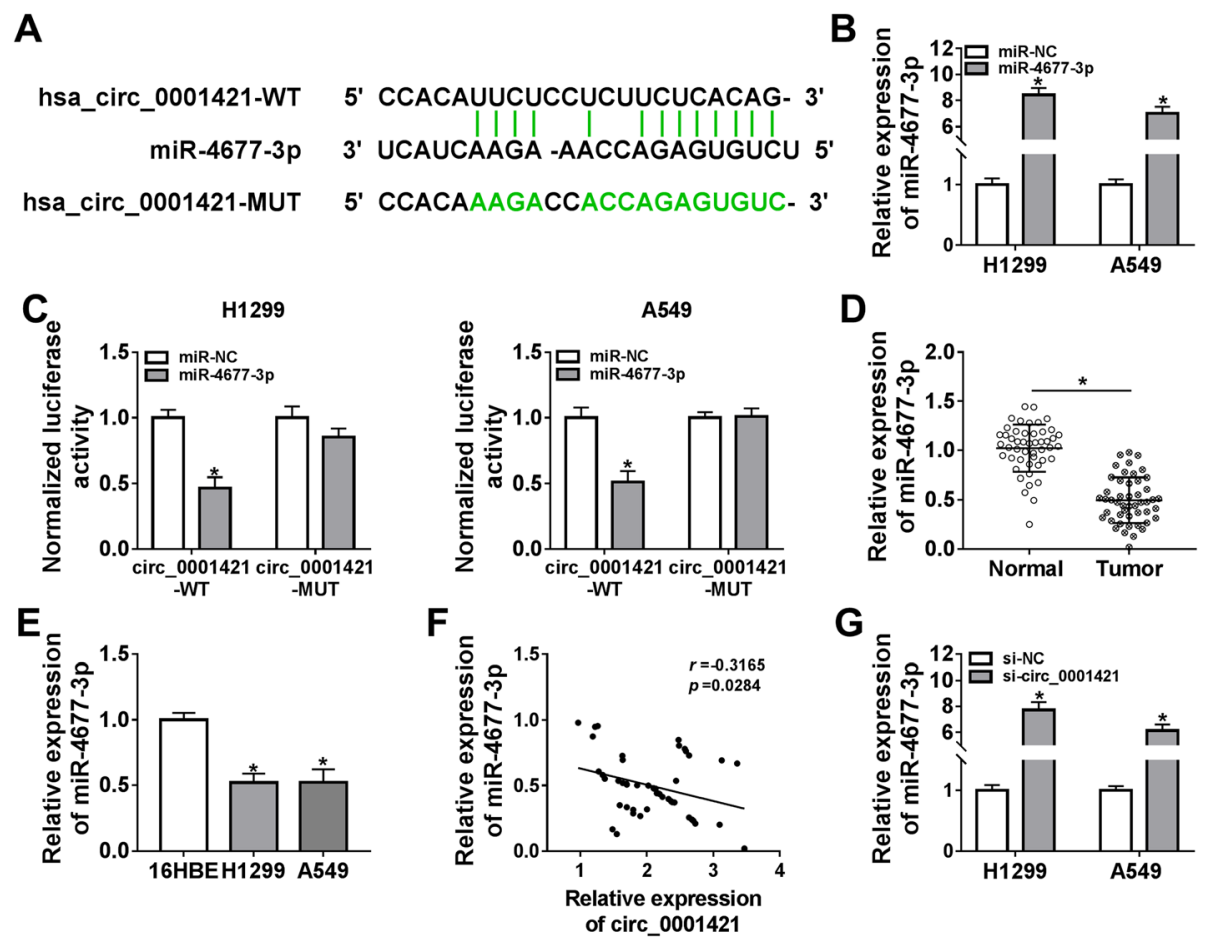

Fig. 3 Circ_0001421 could act as a sponge for miR-4677-3p. a The predicted binding sites between circ_0001421 and miR-4677-3p were shown. b The expression of miR-4677-3p in H1299 and A549 cells transfected with miR-NC or miR-4677-3p was detected by qRT-PCR. (C) Dual-luciferase reporter assay was performed to verify the relationship between circ_0001421 and miR-4677-3p in H1299 and A549 cells. d miR-4677-3p expression in tumor and adjacent normal tissues was examined by qRT-PCR. e qRT-PCR was used to determine miR-4677-3p expression in A549, H1299 and 16HBE cells. f The correlation between circ_0001421 and miR-4677-3p expression was analyzed in LC tissues by Pearson coefficient analysis. $\mathbf{g}$ miR-4677-3p expression in H1299 and A549 cells transfected with si-NC or si-circ_0001421 was measured by qRT-PCR. ${ }^{*} P<0.05$ 
Circ_0001421 regulated LC cell progression by targeting miR-4677-3p in LC cells

To understand whether miR-4677-3p was involved in circ_0001421 regulation of cell biological behaviors, si-circ_0001421 and anti-miR-4677-3p were cotransfected into A549 and H1299 cells. The results showed that miR-4677-3p was inhibited by transfection of anti-miR-4677-3p in A549 and H1299 cells than cells transfected anti-miR-NC (Fig. 4a). MTT and Transwell assay indicated that miR-4677-3p inhibition reversed the inhibition effects of si-circ
0001421 on the proliferation (Fig. 4b), migration (Fig. 4c) and invasion (Fig. 4d) of A549 and H1299 cells. Moreover, glucose consumption and lactate production were decreased by circ_0001421 knockdown and then recovered by miR-4677-3p inhibition knockdown in A549 and H1299cells (Fig. 4e-f), and the inhibition effect of si-circ_0001421 on HK2 expression could be reversed by silencing miR-4677-3p (Fig. 4g). These findings suggested that circ_0001421 executed its carcinogenic role in the progression of LC cells by targeting miR-4677-3p.

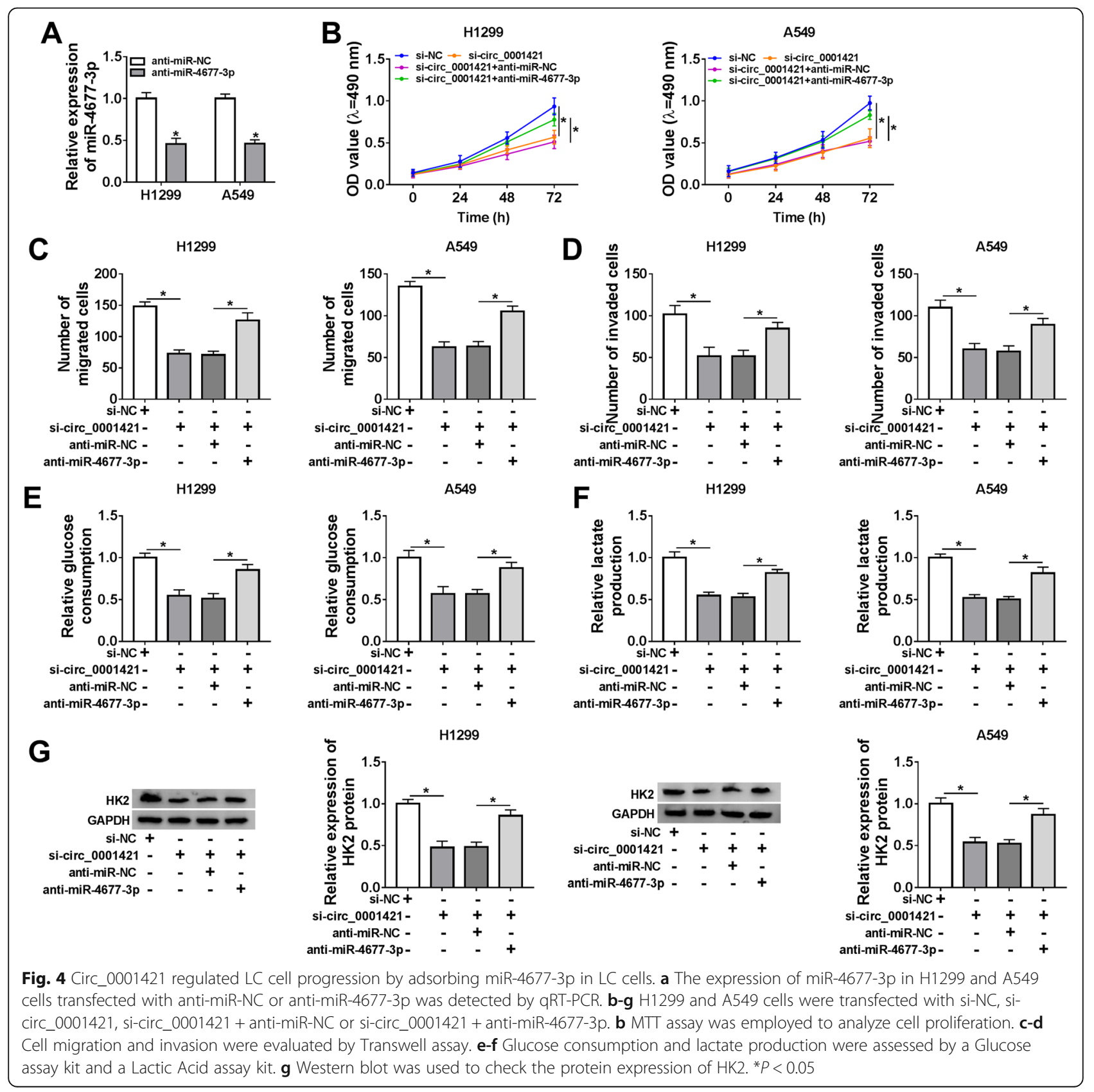


CDCA3 was a target gene of miR-4677-3p in LC

MiRNAs often play biological roles by binding to the 3'UTR of mRNAs [20]. To verify the target mRNAs of miR-4677-3p, Starbase 3.0 was employed. As shown in Fig. 5a, the 3'UTR of CDCA3 contained miR-4677-3p binding sites. To further confirm this prediction, dual-luciferase reporter assay was performed in A549 and H1299 cells. We observed that the luciferase activity of CDCA3-3'UTR-WT was reduced in A549 and H1299 cells transfected with miR-4677-3p, while the luciferase activity of CDCA3-3'UTR-MUT was no obvious variation (Fig. 5b). To explore the role of CDCA3 in LC, CDCA3 expression in LC tissues and cells was detected by western blot. As shown in Fig. 5c-d, CDCA3 protein expression in LC tissues and cells was drastically increased compared to normal tissues and cells. Unsurprisingly, CDCA3 expression was negatively correlated with miR-4677-3p expression and positively related to circ_0001421 level (Fig. 5ef). Besides, miR-4677-3p overexpression reduced the protein expression of CDCA3 in A549 and H1299 cells (Fig. 5g). Western blot results also revealed that circ_0001421 knockdown degraded CDCA3 expression, while this effect could be weakened by miR4677-3p deficiency in A549 and H1299 cells (Fig. 5h-i). Collectively, miR-4677-3p could bind to CDCA3 and regulate its expression in LC.

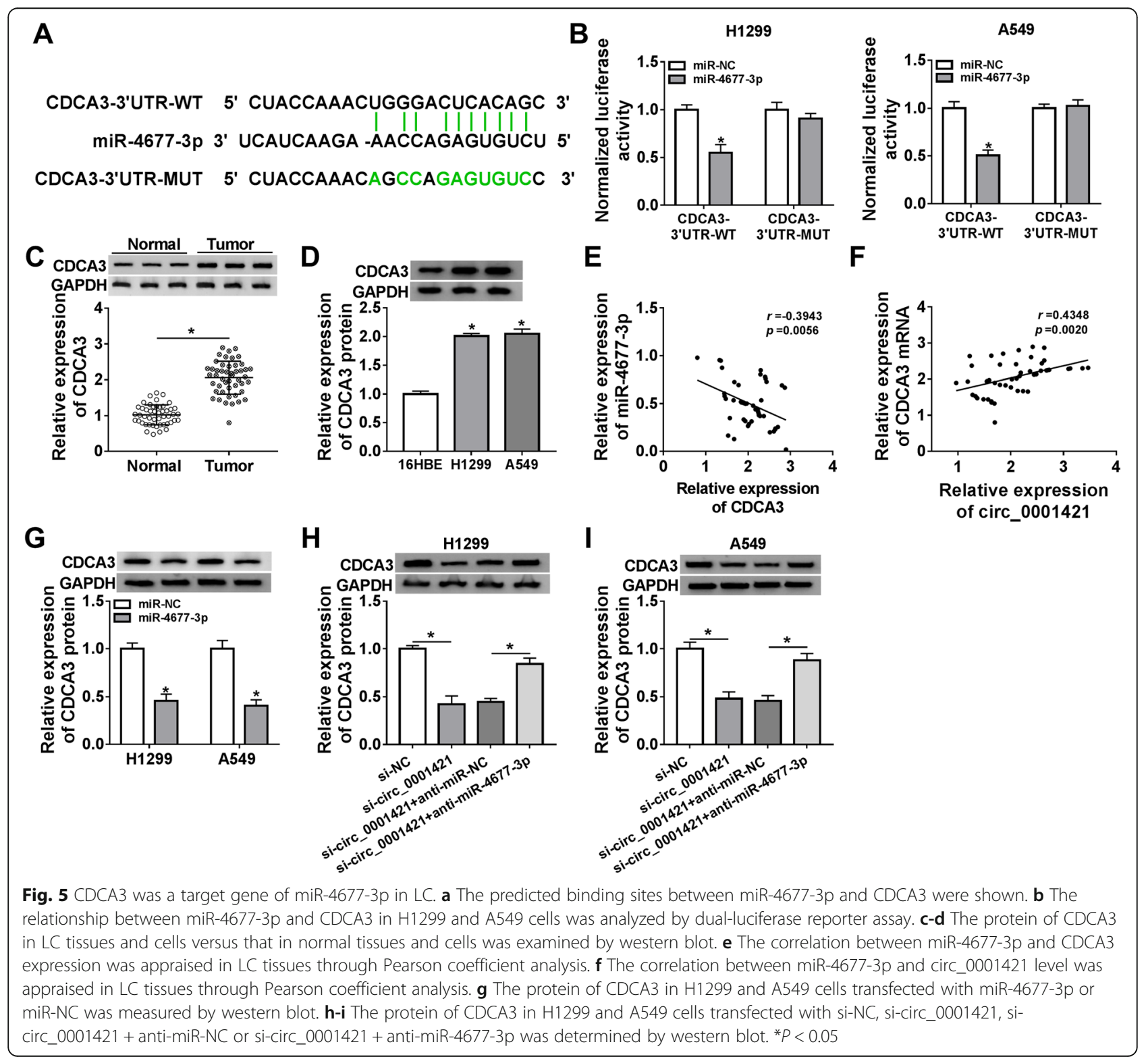


CDCA3 overexpression reversed the inhibitory effects of circ_0001421 knockdown on LC cell proliferation,

migration, invasion and glycolysis

Considering that circ_0001421 could regulate the expression of CDCA3, we then investigated whether circ_0001421 played a role in LC cells by regulating CDCA3. As shown in Fig. 6a, CDCA3 protein expression in A549 and H1299 cells transfected with CDCA3 was significantly increased than the control group. In above data, we demonstrated that circ_0001421 knockdown impeded cell proliferation (Fig. 6b), migration (Fig. 6c) and invasion (Fig. 6d), all of which could be neutralized by overexpression of CDCA3 in A549 and H1299 cells. Meanwhile, CDCA3 overexpression offset the repression of circ_0001421 knockdown on glucose consumption (Fig. 6e), lactate production (Fig. 6f) and the protein of HK2 (Fig. 6g) in A549 and H1299 cells. These results suggested that circ_0001421 could regulate the development of LC cells by regulating CDCA3.

\section{Knockdown of circ_0001421 inhibited cell growth of LC cells in vivo}

To further certify the promotion of circ_0001421 in LC development in vivo, the xenograft experiments were

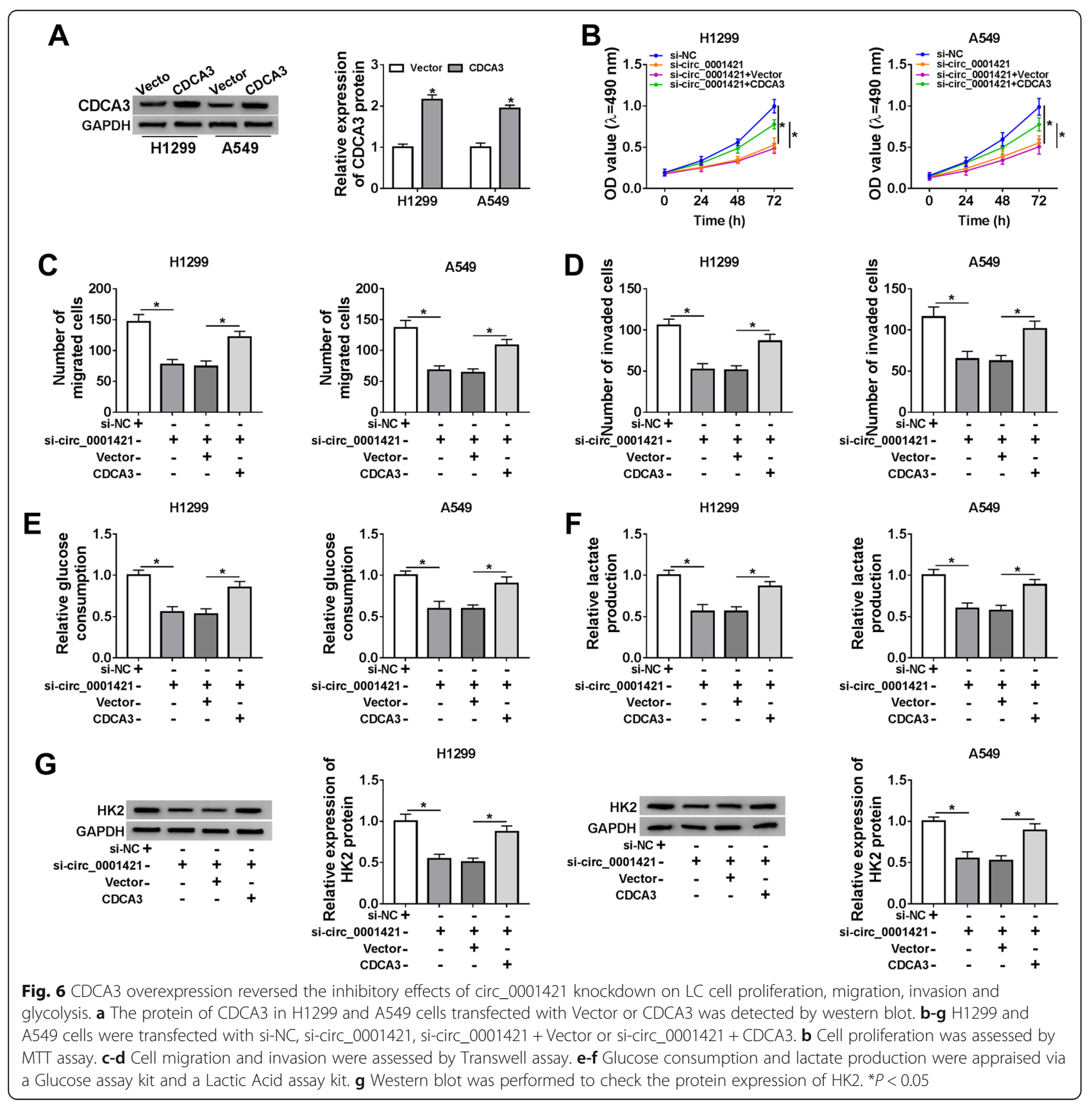




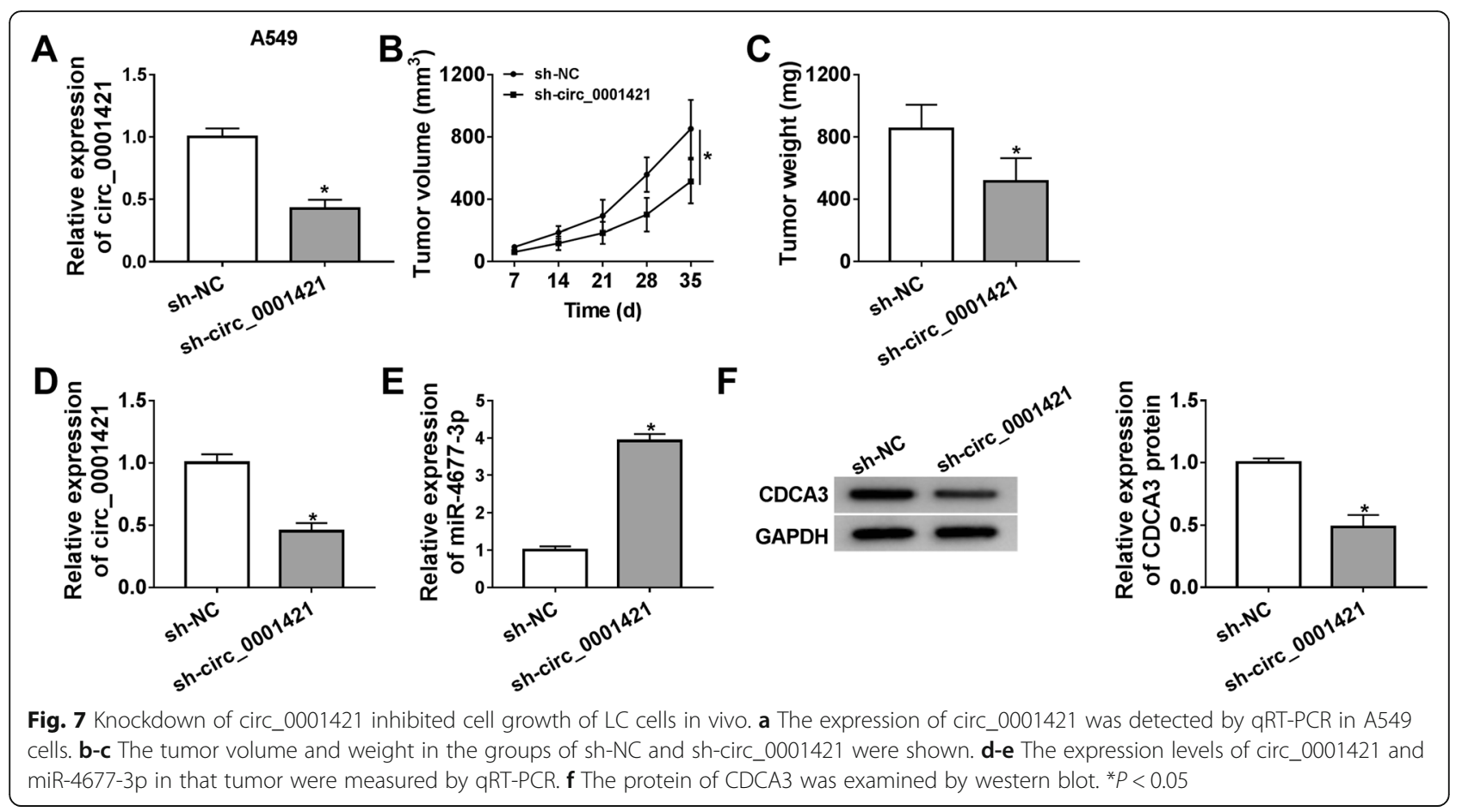

carried out in nude mice. Our data showed that circ 0001421 was downregulated in A549 cells (Fig. 7a). As demonstrated in Fig. 7b-c, the volume and weight of the tumors in sh-circ_0001421 group were smaller than those tumors in sh-NC group. Meanwhile, qRT-PCR indicated that the expression of circ_0001421 was reduced, while miR-4677-3p expression was increased in tumors of sh-circ_0001421 group (Fig. 7d-e). The protein expression of CDCA3 in sh-circ_0001421 group was markedly reduced (Fig. 7f). In summary, these results identified that inhibition of circ_0001421 restrained LC cell growth in vivo.

\section{Discussion}

Despite major breakthroughs in the treatment for LC in recent years, LC still poses a great threat to human health. Many reports demonstrated that abnormal circRNA expression was related to the development of a variety of human cancers, including LC [21, 22]. A previous study claimed that circ_0001421 was up-regulated in NSCLC tissues [12]. In this research, we manifested the high expression of circ_0001421 in LC tissues and cell lines. Furthermore, we explored the functional effects of circ_0001421 and revealed that circ_0001421 deficiency restrained cell proliferation, migration and invasion, and reduced glucose consumption, lactate production and the protein of HK2, suggesting silencing circ_0001421 also inhibited cell glycolysis. The lactate produced during glycolysis could to disrupt the extracellular matrix, leading to the rapid cell proliferation, inhibiting apoptosis, and facilitating cell metastasis [23]. These results elucidated that circ_0001421 might play a role in promoting the development of LC.

Mechanistically, it has been determined that circRNAs functioned as competing endogenous RNAs (ceRNAs) of miRNAs to regulate mRNA expression [24]. For instance, circRNA-002178 served as a ceRNA of miR-34 to promote PDL1 expression in lung adenocarcinoma [25]. Circ-ZKSCAN1 modulated FAM83A expression via sponging miR-330-5p in NSCLC cells [26]. In this paper, miR-4677-3p was a downstream target of circ_0001421. Furthermore, miR-4677-3p expression and role have been mentioned in the study of Han et al., lncRNA LINC02418 elevated proliferation and invasion of NSCL $\mathrm{C}$ cells via sponging miR-4677-3p and regulating SEC61G [27]. As expected, circ_0001421 could negatively modulate miR-4677-3p expression, while interference with miR-4677-3p abrogated the suppressive effects of circ_0001421 knockdown on cell progression, suggesting that miR-4677-3p has an inhibition effect on the development of LC. Taken together, our results confirmed that circ_0001421 contributed to the development of LC by acting as a miR-4677-3p sponge.

The downstream gene of the circ_0001421/miR-4677$3 p$ axis was further explored. CDCA3 was identified as the target of miR-4677-3p by Starbase 3.0 prediction and dual-luciferase reporter assay. A previous report using a microarray data set from 163 tumor types for gene network analysis, demonstrated that CDCA3 was 
prominently up-regulated in breast, lung, and ovarian cancers relative to that normal tissues [28]. In line with these results, we revealed that CDCA3 was enhanced in LC tissues and cells. Additionally, we observed that CDCA3 overexpression inverted the inhibition role of circ_0001421 knockdown in LC cell progression. Furthermore, Adams et al. revealed that high expression of CDCA3 was associated with poor prognosis in patients with NSCLC, and interference with CDCA3 inhibited cell proliferation [29]. Consistent with the results in vitro, circ_0001421 suppression impaired the growth of tumor cells in vivo. Simultaneously, we observed that miR-4677-3p expression was increased, while CDCA3 expression was reduced in the stripped tumor tissues of circ_0001421 decreased group.

\section{Conclusion}

The present study revealed that circ_0001421 participated in cell proliferation, migration, invasion and glycolysis in LC cells via modulating the miR-4677-3p/ CDCA3 axis. The results of this study might provide the meaningful inspiration for exploring LC progression.

\section{Abbreviations}

LC: Lung cancer; circRNAs: Circular RNAs; miR-4677-3p: microRNA-4677-3p; CDCA3: Cell division cycle associated 3; qRT-PCR: quantitative real-time polymerase chain reaction; MTT: Methyl thiazolyl tetrazolium; HK2: Hexokinase 2; ncRNA: non-coding RNA; miRNAs: microRNAs; FBS: Fetal bovine serum; cDNA: complementary DNA; SDS-PAGE: Sodium dodecyl sulfate polyacrylamide gel electrophoresis

\section{Acknowledgements}

Thanks for all participants involved in this study.

\section{Authors' contributions}

$\mathrm{HH}$ designed and supervised the study. KZ and YJ conducted the experiments and drafted the manuscript. LQ conducted the experiments and supervised the study. JX collected and analyzed the data. XJ contributed the methodology and analyzed the data. XC operated the software and edited the manuscript. All authors read and approved the final manuscript.

\section{Funding}

None.

\section{Availability of data and materials}

The data sets used and/or analyzed during the current study are available from the corresponding author on reasonable request.

\section{Ethics approval and consent to participate}

This research was authorized by the Ethics Committee of The Fourth Affiliated Hospital of Nantong University; The First People's Hospital of Yancheng and was carried out according to the guidelines of Declaration of Helsinki.

Animal experimental protocols were permitted by the Animal Care and Use Committee of The Fourth Affiliated Hospital of Nantong University; The First People's Hospital of Yancheng and performed in accordance with the guidelines of the National Animal Care and Ethics Institution.

\section{Consent for publication}

Not applicable.

\section{Competing interests}

The authors declare that they have no competing interest.

\section{Author details}

'Department of Respiratory and Critical Medicine, The Fourth Affiliated Hospital of Nantong University; The First People's Hospital of Yancheng, No.66 Renmin South Road, Yancheng 224000, Jiangsu, China. ${ }^{2}$ Department of Cardiothoracic Surgery, The Fourth Affiliated Hospital of Nantong University; The First People's Hospital of Yancheng, Yancheng 224000, Jiangsu, China. ${ }^{3}$ Department of Pathology, The Fourth Affiliated Hospital of Nantong University; The First People's Hospital of Yancheng, Yancheng 224000, Jiangsu, China.

Received: 1 September 2020 Accepted: 15 October 2020

Published online: 28 October 2020

\section{References}

1. Torre LA, Bray F, Siegel RL, Ferlay J, Lortet-Tieulent J, Jemal A. Global cancer statistics, 2012. CA Cancer J Clin. 2015:65(2):807.

2. Xu K, Liu X, Mao X, Xue L, Wang R, Chen L, et al. MicroRNA-149 suppresses colorectal cancer cell migration and invasion by directly targeting forkhead box transcription factor FOXM1. Cell Physiol Biochem. 2015:35(2):499-515.

3. Hirsch FR, Scagliotti GV, Mulshine JL, Kwon R, Curran WJ Jr, Wu YL, et al. Lung cancer: current therapies and new targeted treatments. Lancet. 2017; 389(10066):299-311

4. Wang $X$, Liu X, Li AY, Chen L, Lai L, Lin HH, et al. Overexpression of HMGA2 promotes metastasis and impacts survival of colorectal cancers. Clin Cancer Res. 2011;17(8):2570-80.

5. Meng S, Zhou H, Feng Z, Xu Z, Tang Y, Li P. CircRNA: functions and properties of a novel potential biomarker for cancer. Mol Cancer. 2017:16(1): 94.

6. Qu S, Yang X, Li X, Wang J, Li H. Circular RNA: a new star of noncoding RNAs. Cancer Lett. 2015;365(2):141-8.

7. Sun J, Qiao Y, Song T, Wang H. MiR-495 suppresses cell proliferation by directly targeting HMGA2 in lung cancer. Mol Med Rep. 2019;19(3):1463-70.

8. Zhang X, Wang S, Wang H, Cao J, Huang X, Chen Z, et al. Circular RNA circNRIP1 acts as a microRNA-149-5p sponge to promote gastric cancer progression via the AKT1/mTOR pathway. Mol Cancer. 2019;18(1):20.

9. Ren S, Liu J, Feng Y, Li Z, He L, Li L, et al. Knockdown of circDENND4C inhibits glycolysis, migration and invasion by up-regulating miR-200b/c in breast cancer under hypoxia. J Exp Clin Cancer Res. 2019:38(1):1-12.

10. Zhang PF, Pei X, Li KS, Jin LN, Wang F, Wu J, et al. Circular RNA circFGFR1 promotes progression and anti-PD-1 resistance by sponging miR-381-3p in non-small cell lung cancer cells. Mol Cancer. 2019;18(1):1-13.

11. Wang Y, Li H, Lu H, Qin Y. Circular RNA SMARCA5 inhibits the proliferation, migration, and invasion of non-small cell lung cancer by miR-19b-3p/ HOXA9 axis. OncoTargets Ther. 2019;12:7055.

12. Jin M, Shi C, Yang C, Liu J, Huang G. Upregulated circRNA ARHGAP10 predicts an unfavorable prognosis in NSCLC through regulation of the miR150-5p/GLUT-1 axis. Mol Ther Nucleic Acids. 2019;18:219-31.

13. Mei J, Xu R, Hao L, Zhang Y. MicroRNA-613: a novel tumor suppressor in human cancers. Biomed Pharmacother. 2020;123:109799.

14. Liang G, Meng W, Huang X, Zhu W, Yin C, Wang C, et al. miR-196b-5pmediated downregulation of TSPAN12 and GATA6 promotes tumor progression in non-small cell lung cancer. Proc Natl Acad Sci U S A. 2020; 117:4347-57.

15. Chen FF, Sun N, Wang $Y, X i$ HY, Yang $Y, Y u$ BZ, et al. miR-212-5p exerts tumor promoter function by regulating the Id3/PI3K/Akt axis in lung adenocarcinoma cells. J Cell Physio. 2020;235:7273-82.

16. Zhong Y, Wang J, Lv W, Xu J, Mei S, Shan A. LncRNA TTN-AS1 drives invasion and migration of lung adenocarcinoma cells via modulation of miR-4677-3p/ZEB1 axis. J Cell Biochem. 2019:120(10):17131-41.

17. Zhang Y, Yin W, Cao W, Chen P, Bian L, Ni Q. CDCA3 is a potential prognostic marker that promotes cell proliferation in gastric cancer. Oncol Rep. 2019;41(4):2471-81

18. Oian W, Zhang Z, Peng W, Li J, Gu Q, Ji D, et al. CDCA3 mediates p21dependent proliferation by regulating E2F1 expression in colorectal cancer. Int J Oncol. 2018:53(5):2021-33.

19. Bi L, Zhou B, Li H, He L, Wang C, Wang Z, et al. A novel miR-375-HOXB3CDCA3/DNMT3B regulatory circuitry contributes to leukemogenesis in acute myeloid leukemia. BMC Cancer. 2018;18(1):182. 
20. Lin X, Qiu W, Xiao Y, Ma J, Zhang K, Yong-Guang G, et al. MiR-199b-5p suppresses tumor angiogenesis mediated by vascular endothelial cells in breast cancer by targeting ALK1. Front Genet. 2019;10:1397.

21. Yao J, Xu G, Zhu L, Zheng H. circGFRA1 enhances NSCLC progression by sponging miR-188-3p. OncoTargets Ther. 2020;13(549):549-58.

22. Zhang L, Guo Y. Silencing circular RNA-ZNF652 represses proliferation and EMT process of renal carcinoma cells via raising miR-205. Artif Cells Nanomed Biotechnol. 2020;48(1):648-55.

23. Kong XZ, Hu SS, Sun Z, Zuo LH, Kang J, Zhu ZF, et al. Regulation of aerobic glycolysis by long non-coding RNAs in cancer. Biochem Biophys Res Commun. 2016:479(1):28-32.

24. Qiu Y, Pu C, Li Y, Qi B. Construction of a circRNA-miRNA-mRNA network based on competitive endogenous RNA reveals the function of circRNAs in osteosarcoma. Cancer Cell Int. 2020;20(1):48.

25. Wang J, Zhao X, Wang Y, Ren F, Sun D, Yan Y, et al. circRNA-002178 act as a ceRNA to promote PDL1/PD1 expression in lung adenocarcinoma. Cell Death Dis. 2020;11(1):1-11.

26. Wang Y, Xu R, Zhang D, Lu T, Yu W, Wo Y, et al. Circ-ZKSCAN1 regulates FAM83A expression and inactivates MAPK signaling by targeting miR-330$5 p$ to promote non-small cell lung cancer progression. Transl Lung Cance Res. 2019;8(6):862-75.

27. Han B. LncRNA LINC02418 regulates proliferation and apoptosis of nonsmall cell lung cancer cells by regulating miR-4677-3p/SEC61G. Euro Rev Med Pharma Sci. 2019;23(23):10354-62.

28. Itzel T, Scholz P, Maass T, Krupp M, Marquardt JU, Strand S, et al. Translating bioinformatics in oncology: quilt-by-profiling analysis and identification of KIF18B and CDCA3 as novel driver genes in carcinogenesis. Bioinformatics. 2015;31(2):216-24.

29. Adams MN, Burgess JT, He Y, Gately K, Snell C, Zhang SD, et al. Expression of CDCA3 is a prognostic biomarker and potential therapeutic target in non-small cell lung cancer. J Thorac Oncol. 2017;12(7):1071-84.

\section{Publisher's Note}

Springer Nature remains neutral with regard to jurisdictional claims in published maps and institutional affiliations.

Ready to submit your research? Choose BMC and benefit from:

- fast, convenient online submission

- thorough peer review by experienced researchers in your field

- rapid publication on acceptance

- support for research data, including large and complex data types

- gold Open Access which fosters wider collaboration and increased citations

- maximum visibility for your research: over $100 \mathrm{M}$ website views per year

At $\mathrm{BMC}$, research is always in progress.

Learn more biomedcentral.com/submissions 